\title{
Current Flow in Upcoming Microelectronic Devices
}

\author{
Viktor Sverdlov, Hans Kosina, and Siegfried Selberherr \\ Institute for Microelectronics, Technische Universität Wien \\ Gusshausstrasse 27-29, \\ A-1040 Vienna, Austria \\ Email: \{sverdlov, kosina, selberherr\}@iue.tuwien.ac.at
}

\begin{abstract}
An overview of models used for the simulation of current transport in upcoming microelectronic devices within the framework of TCAD applications is presented. Modern enhancements of semiclassical transport models based on microscopic theories as well as quantum mechanical methods used to describe coherent and dissipative quantum transport are specifically addressed. This comprises the incorporation of quantum correction and tunneling models up to dedicated quantum-mechanical simulators, and mixed approaches accounting for both, quantum interference and scattering. Specific TCAD requirements are discussed from an engineer's perspective and an outlook on future research directions is given.
\end{abstract}

\section{INTRODUCTION}

The breathtaking increase in computational power and speed of integrated circuits in the past decades has been supported by the aggressive size reduction of semiconductor devices. This trend is expected to continue in the coming decade as predicted and institutionalized by the International Technology Roadmap for Semiconductors [1]. Today, when the $90 \mathrm{~nm}$ technology node with physical transistor gate lengths in the range of $40 \mathrm{~nm}$ is in mass production, the challenge is to introduce the $65 \mathrm{~nm}$ technology node already within a year. A new technology node is introduced every 3 years, with a long-term projection of the $22 \mathrm{~nm}$ node to be in mass production by the year 2016. A possibility to build metal-oninsulator field effect transistors (MOSFETs) with even shorter gate lengths has been successfully shown after the $6 \mathrm{~nm}$ gate length transistor has been demonstrated in research labs [2], [3]. From a theoretical point of view even a few nm gate length device has been predicted to be functional [4], [5]. Nevertheless, emerging outstanding technological challenges related to different aspects of MOSFET fabrication and reliability in mass production, as well as the rapidly increasing power dissipation may slow down the so far exponential scaling of Complimentary MOSFETs (CMOS). Besides, with the ongoing search for new technological solutions vital for CMOS downscaling, developing conceptually new devices and architectures is becoming increasingly important. New nanoelectronic structures, such as carbon nanotubes, nanowires, and molecules, are considered to be the most prominent candidates for the post-CMOS era. Since conventional MOSFETs are already operating in the sub-100 $\mathrm{nm}$ range, new nanoelectronic devices are expected to complement and substitute some of the

This research was supported by the Austrian Science Fund FWF, project P17285-N02, and by the European Commission, project SINANO IST-506844 current CMOS functions after being integrated into CMOS technology.

Technology computer-aided design (TCAD) tools are used to assist in development and engineering at practically all stages ranging from process simulation to device and circuit optimization. The main purpose of TCAD is the technologydevelopment related cost reduction which is estimated to about $40 \%$ according to ITRS [1]. Due to the aggressive downscaling of CMOS device feature sizes and newly emerging nanoelectronic devices, various shortcomings of presently applied TCAD tools appear. These tools are frequently based on semiclassical macroscopic transport models. From an engineering point of view, classical models like the drift-diffusion model, have enjoyed an amazing success due to their relative simplicity, numerical robustness, and the ability to perform two- and three-dimensional simulations on large unstructured grids [6]. Hot-carrier effects have motivated the development of higherorder transport models such as the hydrodynamic, energytransport and six-moments models [7]. However, inaccuracies originate from the non-local nature of carrier propagation in ultra-scaled devices [8].

Non-local effects may be of classical or quantummechanical nature, depending on the underlying physics relevant to the transport process. Classical non-localities appear when the mean-free path is comparable to the device feature size. Quantum mechanical non-local effects start to determine the transport properties when the devices' size is of the order of the De-Broglie electron wave length. Size quantization of carrier motion in inversion layers of MOSFETs and in ultrascaled multi-gate devices as well as the tunneling current, including the gate leakage current, are the most important examples of quantum effects in MOSFETs.

Fig. 1 shows the hierarchy and mutual interrelation of models currently used for the description of current transport. Semiclassical transport models are based on the Boltzmann equation which includes scattering integrals describing realistic microscopic processes. These semiclassical models, augmented with quantum corrections, are still of great importance due to their relative computational simplicity, numerical stability, and an ability to provide reasonable quantitative results within seconds even for devices with gate length as short as $50 \mathrm{~nm}$. A brief overview of the currently developed semiclassical transport models will be presented in Section 2.

Quantum ballistic transport models describe a coherent propagation of carriers. They are based on the solution of the Schrödinger equation for the wave function, supplemented 


\section{Quantum Ballistic Transport}

Wave Function / Schrödinger Equation

\section{Semiclassical Transport}

Distribution Function / Boltzmann Equation

\begin{tabular}{|c|}
\hline Quantum Transport \\
\hline Density Matrix / von Neumann Equation \\
\hline NEGF / Dyson Equation \\
\hline Wigner Function / Wigner Equation \\
\hline
\end{tabular}

Fig. 1. Schematic classification of approaches used in semiconductor current transport modeling.

with the corresponding boundary conditions. This approach is efficient and provides accurate results when carrier scattering is irrelevant and can be neglected. The method will be illustrated in Section 3 with an example of transport in carbon nanotubes [9].

Finally, dissipative quantum transport theory represents the most complete description of transport, which combines the coherent carrier motion between the scattering events with coherence (or phase) breaking due to carrier scattering. Different formalisms are currently used, based on the Dyson equation for the non-equilibrium Green's functions, the Liouville/VonNeumann equation for the density matrix, or the Wigner transport equation. Section 4 deals with quantum transport characterized by both scattering and quantization. A conclusion will summarize the main findings and give directions for future research.

\section{SEMICLASSICAL TRANSPORT}

After the ground-breaking work of Scharfetter and Gummel [10], who first proposed a robust discretization scheme for the drift-diffusion equation, computer programs like MINIMOS [11] and PISCES [12] played a pioneering role in numerical simulation of current transport properties of semiconductor devices. Since then, numerous transport models of increasing complexity have been introduced. The semiclassical transport description is based on the Boltzmann equation for the distribution of carriers $f(\mathbf{r}, \mathbf{k}, t)$ in the phase space. The Boltzmann equation includes carriers' scattering with phonons, impurities, interfaces, and other scattering sources through the corresponding collision integrals. Although the solution of the Boltzmann equation can be found numerically by means of Monte Carlo (MC) methods, TCAD models based on moments of the distribution function are highly desirable. Being computationally significantly less expensive than the MC method, these higher-order moments' methods provide a reasonable quantitative answer for devices as short as $50 \mathrm{~nm}$ within seconds. The fairly new six moments model [7] based on non-Maxwellian distribution takes naturally into account the hot-carrier effects such as avalanche generation, hot carrier induced gate currents, or hot-carrier diffusion, which typically take place in Silicon-On-Insulator (SOI) floating body MOSFETs. For the purpose of calibration the full-band MC method is often accepted, since it can precisely account for the various scattering processes [13].

Another important development of transport models is related to the MC methods for solving the Boltzmann equation. After the pioneering work of Kurosawa in 1966 [14], who was the first to apply the MC method to simulate carrier transport in semiconductors, the significantly improved MC method was successfully applied to transport description in a variety of semiconductors [15]. For electrons in silicon, the most thoroughly investigated case, it is believed that a satisfactory understanding of the band structure and of the basic scattering mechanisms has been achieved giving rise to a "standard model" [16]. Nowadays, an accurate MC evaluation of carrier transport properties in inversion layers is of primary importance for predicting performance of modern CMOS bulk devices. Due to the strong confinement of carriers in the inversion layer of bulk MOSFETs or due to the geometric confinement in multi-gate FETs the carrier motion is quantized in one or two confinement directions giving rise to the formation of subbands. One possibility to address the effect of quantum confinement on the electron concentration is to use an effective potential. This can be achieved by a convolution of the electrostatic potential with a Gaussian function, which leads to a smoothing of the original potential [17], [18], [19]. Another option is to use the self-consistent Poisson-Schrödinger-based quantum corrected potential [20], [21], which suppresses the carrier concentration close to the interface, mimicking the real quantum-mechanical behavior. These approaches combine advantages of full-band structure and flexibility of scattering processes of three-dimensional classical MC simulations [22] with the generality of material composition and transport peculiarities due to quantum confinement and may also address the strain effects [23]. The MC approach may incorporate the quantized carrier motion in the direction orthogonal to the current exactly. The quantum-mechanical motion of carriers in the confined direction is addressed by the self-consistent solution of the corresponding Schrödinger and Poisson equation, leading to the formation of subbands. The carrier motion within each subband may still be considered semiclassical and therefore can be well described by the corresponding Boltzmann equation written for the subband distribution function $f_{n}(\mathbf{r}, \mathbf{k}, t)$. Because of possible carrier transitions between 


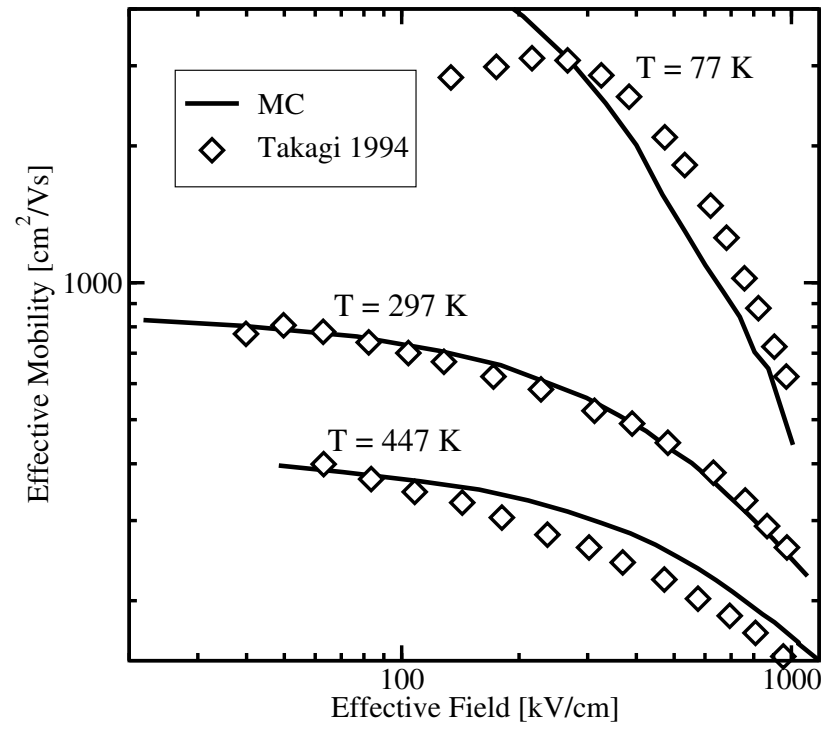

Fig. 2. Comparison of subband MC simulations with the experimental [24] universal mobility of surface layer in silicon. The deviation of the experimental mobility from simulations at low effective fields is due to Coulomb scattering not included in the MC simulations.

different subbands due to scattering, the collision integrals on the right-hand-side of the Boltzmann equation should include the terms responsible for the intersubband scattering processes. The transport in the inversion layer of a MOSFET is finally described by a set of Boltzmann equations for every subband, coupled to each other via the intersubband scattering integrals. The set of the subband Boltzmann equations for $f_{n}(\mathbf{r}, \mathbf{k}, t)$ is conveniently solved by a MC method. This approach therefore combines the advantages of a quantum description in confinement direction with a semiclassical description in transport direction and represents a transition between semiclassical and quantum-mechanical pictures. An example of the simulation of the low-field surface mobility in inversion layers of silicon, when the transport in the current direction may be treated semiclassically is shown in Fig. 2, together with the experimental "universal mobility" curve [24]. In order to reproduce the universal mobility curve, up to 40 unprimed and 20 primed subbands formed at a (100) silicon interface were taken into account, with realistic electron-phonon and surface roughness scattering included [25].

\section{QUANTUM-BALLISTIC TRANSPORT}

With the aggressive downscaling of MOSFET dimensions continuing, the classical description of carrier motion in transport direction is gradually losing its validity. When the characteristic scale of the potential variation along the channel is comparable to the De-Broglie wave length of a carrier, a TCAD transport model must include the quantum effects in transport direction. If scattering processes can be ignored and particle propagation in the device is ballistic, the carrier motion is determined by the solution of the Schrödinger equation, supplemented with open boundary conditions [26][28]. In order to determine the current density, it suffices

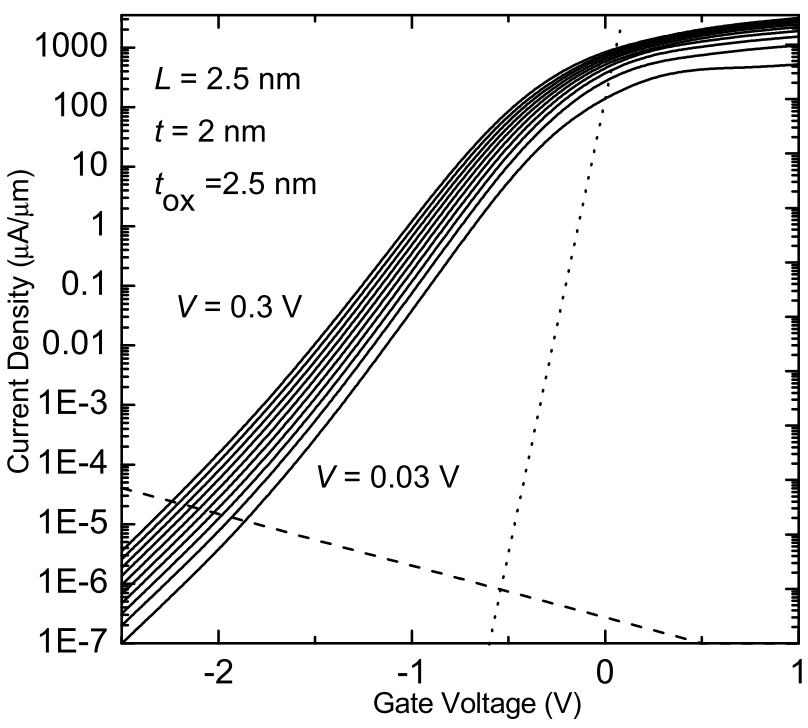

Fig. 3. Subthreshold characteristics for a double-gate MOSFET with silicon thickness of $2 \mathrm{~nm}$, gate length of $2.5 \mathrm{~nm}$ and the oxide thickness of $2.5 \mathrm{~nm}$ [4] The dotted line corresponds to the ideal $60 \mathrm{mV} /$ decade subthreshold slope. The dashed line shows the leakage current.

to know the transmission coefficient $T C(\mathcal{E})$ as well as the supply function $N\left(\mathcal{E}_{x}\right)$ from the electrodes [29]. A similar approach can also be used to determine the gate leakage current [30]. The solution of the Schrödinger equation with open boundary conditions can be achieved by means of the quantum transmitting boundary method [31], [32]. An established alternative framework for these calculations is the non-equilibrium Green's Function method [33] in its reduced coherent version. It is conveniently used for onedimensional studies of resonant tunneling diodes or carbon nanotubes. Simulators accounting for a full two-dimensional solution of the open-boundary Schrödinger equation have been reported and applied to the simulation of $10 \mathrm{~nm}$ double-gate MOSFETs [26], [34]. It may appear that in the quantumballistic case the determination of the full wave function as a solution of the Schrödinger equation is not necessary and the knowledge of the transmission coefficient is enough for the current calculations. In the contact block reduction method [35] the transmission function is fully determined by the reduced contact part of the full Green's function. However, the carrier concentration alters the electrostatic potential in the device via the Poisson equation. The carrier concentration is proportional to the square of the wave function, implying that the accurate determination of the transmission coefficient and therefore the current requires a self-consistent solution of the Schrödinger and Poisson equation simultaneously. For quasione-dimensional transport this can be achieved straightforwardly [4]. An example of the output characteristics simulated for an ultra-thin body double-gate MOSFET with a gate length $L$ as short as $2.5 \mathrm{~nm}$ is shown in Fig. 3. Surprisingly, even such a small device possesses an $I_{\text {on }} / I_{\text {off }}$ ratio sufficient for logic applications and displays a reasonable short-channel effect and acceptable DIBL, a conclusion recently reached from 


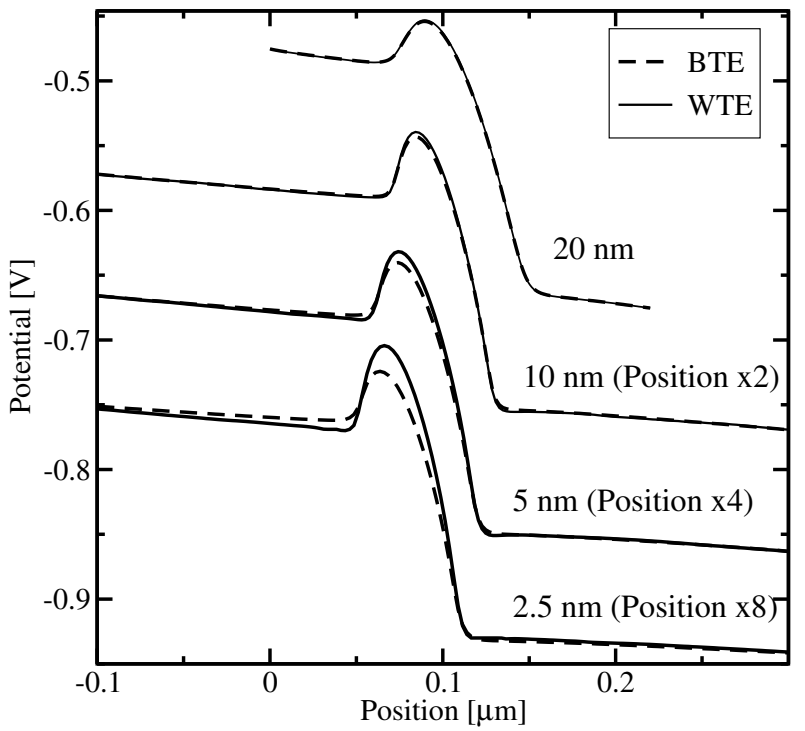

Fig. 4. Self-consistent potential profiles calculated for $n-i-n$ structure with Wigner (solid lines) and Boltzmann (dashed lines) transport equations. For long $n-i-n$ structures results are similar. For short $n-i-n$ structures additional charge due to tunneling electrons results in significantly higher potential barrier.

more detailed atomistic calculations [5]. It should be noted that the sensitivity to small MOSFET dimension variations, control of doping as well as the whole manufacturing process development represent significant challenges for multi-gate MOSFETs with gate length below $10 \mathrm{~nm}$.

The ballistic quantum transport description is justified if the size of the channel region is shorter than the scattering length. In carbon nanotubes, where elastic scattering can be ignored and inelastic scattering has little effect on current [36], the measured current value [37] can well be predicted within the quantum-ballistic approach [9]. Similar methods can be applied to describe the output characteristics of FinFETs in the ballistic approximation [38]. In silicon MOSFETs, however, the mean-free path in the area close to the potential maximum at $300 \mathrm{~K}$ is only a few $\mathrm{nm}$ [39], and the full quantum description which includes dissipative processes must be adopted to simulate MOSFETs with a gate length of around $10 \mathrm{~nm}$. A consistent introduction of realistic scattering into simulators based on the coherent description alone creates outstanding computational difficulties ranging from a necessity to invert huge matrices in NEGF formalism [33] to calculations of nonlocal scattering rates in Pauli master equation approaches [40]. Besides the difficulties of introducing scattering into the simulators based on the coherent description, these simulators are often limited to specific geometries, grids and short length scales, which makes their integration into modern engineering TCAD tools problematic. Nevertheless, these simulation approaches are necessary for the estimation of upper bounds of current transport at the quantum limit.

\section{Dissipative QuANTUM TRANSPORT}

The methods described so far are either based on the assumption of semiclassical or pure quantum-mechanical bal-

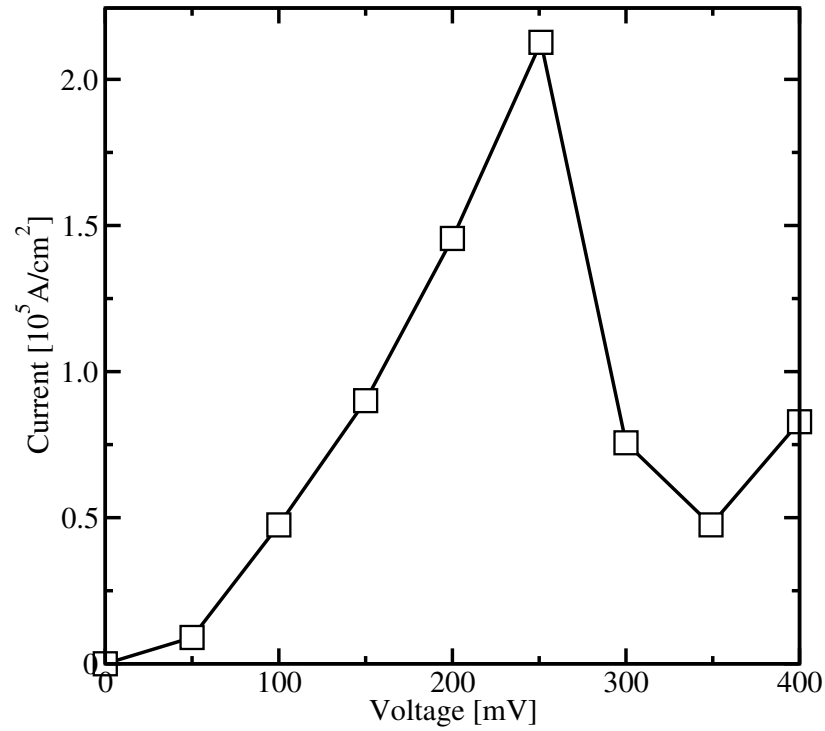

Fig. 5. Typical $I V$ curve of resonant tunneling diode computed with Wigner MC self-consistently. The negative differential resistance after the peak is characteristic for resonant structures.

listic transport. The former modeling approach has proven to be adequate to describe transport in previous generations of microelectronic devices. The latter one may be used for transport description when the carrier coherence length is larger than the devices size. Recent studies show that even for devices with the channel length as short as $15 \mathrm{~nm}$ scattering will still play a significant role [41] and therefore determine the current, in accordance with estimations of the mean-free path in MOSFET structures [39]. Crossover from diffusive to ballistic transport in Si nanowire transistors occurs at approximately $2 \mathrm{~nm}$ [42], a much shorter distance than previously anticipated. An adequate transport model for ultra-scaled MOSFETs must therefore account for quantum mechanical and dissipative effects simultaneously. In modern microelectronic devices quantum effects are usually dominant in a small active region connected to relatively large, heavily doped contact areas where the carrier dynamics is essentially classical. Therefore, TCAD simulators should also be able to incorporate both semiclassical and (dissipative) quantummechanical modeling approaches within the same formalism. To a certain extent, various quantum corrections can serve the purpose, as already discussed.

The non-equilibrium Green's functions method addresses the problem in the most consistent and complete way. Due to its completeness, the method is computationally complex and usually applied to one-dimensional problems [33] and for a restricted set of scattering mechanisms [43] only. The carbon nanotube (CNT) FET, which is widely considered to be a potential alternative to conventional MOSFETs, represents a good example where the nonequilibrium Green's function method provides accurate results [44].

An alternative approach which can handle both quantummechanical and dissipative scattering effects is based on the Wigner function formalism. Realistic scattering processes can 
be easily embedded into the Wigner equation via Boltzmannlike scattering integrals, which turns out to be a good approximation. The Wigner function approach reduces to a semiclassical transport description for slow varying potential providing the important advantage of a seamless treatment between classical and quantum-mechanical regions in device simulations [45]. By applying the method of moments to the equation for the Wigner function, the quantum drift-diffusion or quantum hydrodynamic models can be derived [46]. These models are more convenient for the implementation in TCAD device simulators than a Schrödinger-Poisson solver which strongly depends on non-local quantities. However, while the carrier concentration in the inversion layer of a MOSFET is reproduced correctly within this approach, the method was reported to give improper results for tunneling currents [47].

By analogy to the Monte Carlo methods used for the Boltzmann transport equation, it is tempting to try to solve the quantum Wigner transport equation by means of the MC technique. Such a program was recently realized in [45], [48], [49]. However, since the kernel of the quantum scattering operator is not positively defined, the numerical weight of a particle trajectory increases rapidly, and the numerical stability of a trajectory-based MC algorithm becomes a critical issue. A multiple trajectories method was suggested [45] to overcome this difficulty. In the algorithm developed, the problem of a growing statistical weight of a single trajectory is addressed by creating an increasing number of trajectories with constant weights, which may assume positive and negative values. Being formally equivalent to the former method, the algorithm allows the annihilation of particles with similar statistical properties, introducing a possibility to control the number of trajectories.

The method was recently applied to double-gate MOSFETs [49]. An example of self-consistent potentials for $n-i-n$ Si structures with an intrinsic region of length $W$ ranging from $20 \mathrm{~nm}$ to $2.5 \mathrm{~nm}$, as calculated with Wigner and classical MC is shown in Fig. 4. The doping profile is assumed to increase gradually from the intrinsic channel to the highly doped contacts value over the same distance $W$. Electron-phonon and Coulomb scattering were included. As expected, for thick $W$ the classical and quantum calculations yield similar results for the self-consistent potential. For $W=2.5 \mathrm{~nm}$ an extra space charge due electrons tunneling under the barrier becomes important, which results in the potential barrier increase. In spite the potential barrier increase, the current in self-consistent Wigner simulations was approximately $20 \%$ higher compared to its classical value found by a self-consistent solution of the Boltzmann and the Poisson equations.

The Wigner function method gives accurate results not only for single-barrier devices, but can also be applied to purely quantum-mechanical systems such as resonant tunneling diodes [45]. A typical output characteristic of a GaAs resonant tunneling diode is shown in Fig. 5. Scattering with polar optical phonons as well as Coulomb scattering in the contacts is considered. A region of negative differential resistance common to transport via a resonant level is clearly visible after the resonance peak at $250 \mathrm{mV}$ applied voltage.

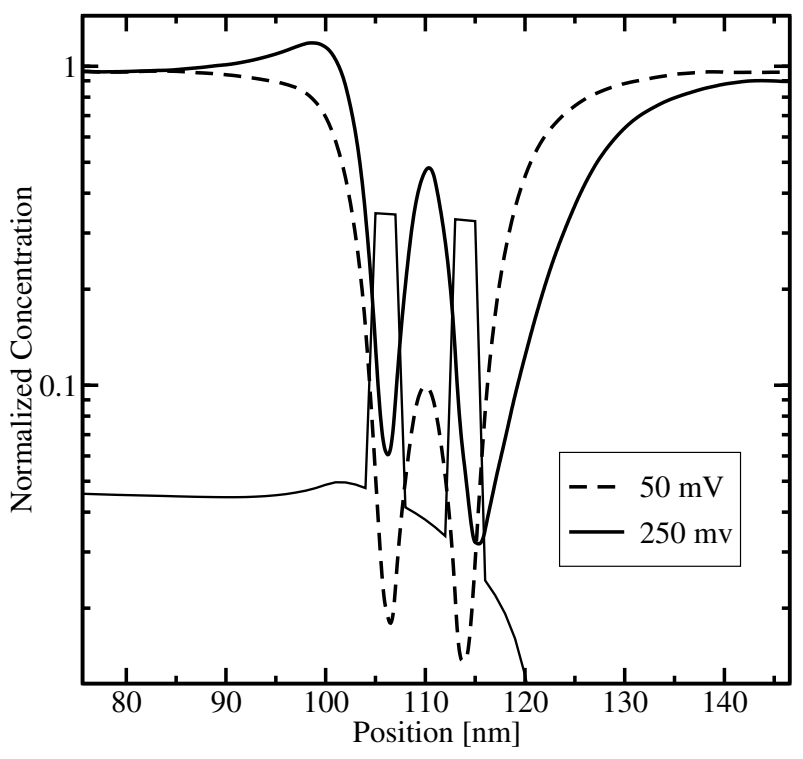

Fig. 6. Normalized electron concentration off-resonance (dashed line) and at resonance (solid line) in double barrier structure. Space charge accumulation in the potential well is seen at the resonance conditions.

Self-consistent solution of the Wigner transport and Poisson equation is mandatory for the correct determination of the resonance position due to charge accumulation at the cathode side of the resonant tunneling diode. A typical distribution of the concentration in resonance condition and off-resonance is shown in Fig. 6. The amount of charge localized in the potential well is much higher at resonance as compared to off-resonance conditions, in accordance with previous simulations [45]. This example demonstrates the importance of quantum mechanical effects for simulations of properties of ultra-scaled devices. It also shows that space charge effects are of crucial importance for the accurate prediction of output characteristics of single- and double-barrier devices.

\section{CONCLUSIONS}

Well established classical TCAD tools are gradually losing their ability to predict accurately the characteristics of nanoscale devices, prompting for enhancement to meet the engineering demands. Classical models using higher moments are able to account for the hot-carrier effects and can reproduce closely results of full-band Monte Carlo methods. Relevant quantum corrections may be incorporated into the Monte Carlo simulators allowing to approximately account for some quantum phenomena.

Full quantum description is required for nanoscale devices. Contrary to carbon nanotubes, where the transport properties can be predicted within the coherent picture, a dissipative quantum description is required for transport calculations in ultra-scaled MOSFETs with gate lengths ranging below $10 \mathrm{~nm}$. One promising option is the Wigner function approach which combines the advantages of a quantum description with accurate scattering models. All quantum-mechanical models must be adapted for engineering applications for which timely results are often more valuable than accurate analyses. 


\section{REFERENCES}

[1] "International Technology Roadmap for Semiconductors - 2005 Edition," 2005, http://www.itrs.net/Common/2005ITRS.

[2] B. Doris, M. Ieong, T. Kanarsky, Y. Zhang, R. A. Roy, O. Documaci, Z. Ren, F.-F. Jamin, L. Shi, W. Natzle, H.-J. Huang, J. Mezzapelle, A. Mocuta, S.Womack, M. Gribelyuk, E. C. Jones, R. J. Miller, H.-S. P. Wong, and W. Haensch, "Extreme Scaling with Ultra-Thin Si Channel MOSFETs," in IEDM Techn. Dig., 2002, pp. 267-270.

[3] H. Iwai, "CMOS Downsizing Toward Sub-10 nm," Solid-State Electron. vol. 48, no. 4, pp. 497-503, 2004.

[4] V. Sverdlov, T. Walls, and K. Likharev, "Nanoscale Silicon MOSFETs A Theoretical Study," IEEE Trans.Electron Devices, vol. 50, no. 9, pp. 1926-1933, 2003.

[5] L. Risch, "Pushing CMOS Beyond the Roadmap," in Proc. European Solid-State Device Research Conf., 2005, pp. 63-68.

[6] S. Selberherr, Analysis and Simulation of Semiconductor Devices. Springer, 1984.

[7] T. Grasser, T.-W. Tang, H. Kosina, and S. Selberherr, "A Review of Hydrodynamic and Energy-Transport Models for Semiconductor Device Simulation," Proc.IEEE, vol. 91, no. 2, pp. 251-274, 2003.

[8] F. Heinz, F. Bufler, A. Schenk, and W. Fichtner, "Quantum Transport Phenomena and Their Modeling," in Symposium on Nano Device Technology, Hsinchu, Taiwan, 2004, pp. 2-8.

[9] M. Pourfath, A. Gehring, E. Ungersböck, H. Kosina, S. Selberherr, B.-H. Cheong, and W. Park, "Separated Carrier Injection Control in Carbon Nanotube Field-Effect Transistors," J.Appl.Phys., vol. 97, no. 10, pp. $1061031-1061033,2005$.

[10] D. Scharfetter and H. Gummel, "Large-Signal Analysis of a Silicon Read Diode Oscillator," IEEE Trans.Electron Devices, vol. 16, no. 1, pp. 64-77, 1969.

[11] S. Selberherr, W. Fichtner, and H. Pötzl, "MINIMOS - A Program Package to Facilitate MOS Device Design and Analysis," in Numerical Analysis of Semiconductor Devices and Integrated Circuits, B. T. Browne and J. J. Miller, Eds., vol. I. Dublin: Boole Press, 1979, pp. 275-279

[12] M. R. Pinto, PISCES IIB. Stanford University, 1985

[13] M. Fischetti and S. Laux, "Monte Carlo Analysis of Electron Transport in Small Semiconductor Devices Including Band-Structure and SpaceCharge Effects," Physical Review B, vol. 38, no. 14, pp. 9721-9745, 1988.

[14] T. Kurosawa, "Monte Carlo Calculation of Hot Electron Problems," in Proc. Intl. Conf. on Physics of Semiconductors, 1966, pp. 424-426.

[15] C. Jacoboni and L. Reggiani, "The Monte Carlo Method for the Solution of Charge Transport in Semiconductors with Applications to Covalent Materials," Reviews of Modern Physics, vol. 55, no. 3, pp. 645-705, 1983.

[16] M. Fischetti and A. Laux, "Monte Carlo Simulation of Electron Transport in Si: The First 20 Years," in 26th European Solid State Device Research Conference, 1996, pp. 813-820.

[17] Y. Li, T.-W. Tang, and X. Wang, "Modeling of Quantum Effects for Ultrathin Oxide MOS Structures with an Effective Potential," IEEE Trans. Nanotechnology, vol. 1, no. 4, pp. 238-242, 2002.

[18] K. Ahmed, P. Kraus, C. Olsen, and F. Nouri, "On the Evaluation of Performance Parameters of MOSFETs with Alternative Gate Dielectrics,' IEEE Trans.Electron Devices, vol. 50, no. 12, pp. 2564-2567, 2003.

[19] P. Palestri, S. Eminente, D. Esseni, C. Fiegna, E. Sangiorgi, and L. Selmi, "An Improved Semi-Classical Monte-Carlo Approach for Nano-Scale MOSFET Simulation," Solid-State Electron., vol. 49, pp. 727-732, 2005.

[20] G. Kathawala, B. Winstead, and U. Ravaioli, "Monte Carlo Simulations of Double-gate MOSFETs," IEEE Trans.Electron Devices, vol. 50 , no. 12, pp. 2467-2473, 2003.

[21] X.-F. Fan, X. Wang, B. Winstead, L. Register, U. Ravaioli, and S. Banerjee, "MC Simulation of Strained-Si MOSFET With Full-Band Structure and Quantum Corrections," IEEE Trans.Electron Devices, vol. 51, no. 6 , pp. 962-970, 2004.

22] D. Ferry, R. Akis, and D. Vasileska, "Quantum Effects in MOSFWETs: Use of an Effective Potential in 3D Monte Carlo Simulations in UltraShort Channel Devices," in IEDM Tech. Dig., 2000, pp. 287-290.

[23] B. Ghosh, X.-F. Fan, L. Register, and S. Banerjee, "Monte Carlo Study of Strained Germanium Nanoscale Bulk pMOSFETs," IEEE Trans.Electron Devices, vol. 53, no. 3, pp. 533-537, 2006.

[24] S.-I. Takagi, A. Toriumi, M. Iwase, and H. Tango, "On the Universality of Inversion Layer Mobility in Si MOSFET's: Part I - Effects of Substrate Impurity Concentration," IEEE Trans.Electron Devices, vol. 41, no. 12, pp. 2357-2362, 1994.
[25] E. Ungersböck and H. Kosina, "The Effect of Degeneracy on Electron Transport in Strained Silicon Inversion Layer," in Proc. Intl. Conf. on Simulation of Semiconductor Processes and Devices, 2005, pp. 311-314.

[26] S. Laux, A. Kumar, and M. Fischetti, "Ballistic FET Modeling Using QDAME: Quantum Device Analysis by Modal Evaluation," IEEE Trans. Nanotechnology, vol. 1, no. 4, pp. 255-259, 2002.

[27] F. Heinz, A. Schenk, A. Scholze, and W. Fichtner, "Full Quantum Simulation of Silicon-on-Insulator Single-Electron Devices," J.Computational Electronics, vol. 1, no. 1, pp. 161-164, 2002.

[28] J. Wang, E. Polizzi, A. Ghosh, S. Datta, and M. Lundstrom, "Theoretical Investigation of Surface Roughness Scattering in Silicon Nanowire Transistor," J.Appl.Phys., vol. 87, pp. 0431 011-0 431 013, 2005.

[29] R. Tsu and L. Esaki, "Tunneling in a Finite Superlattice," Appl.Phys.Lett., vol. 22, no. 11, pp. 562-564, 1973.

[30] A. Gehring, "Simulation of Tunneling in Semiconductor Devices," Dissertation, Technische Universität Wien, 2003.

[31] C. Lent and D. Kirkner, "The Quantum Transmitting Boundary Method,' J.Appl.Phys., vol. 67, no. 10, pp. 6353-6359, 1990.

[32] W. Frensley, "Numerical Evaluation of Resonant States," Superlattices \& Microstructures, vol. 11, no. 3, pp. 347-350, 1992.

[33] R. Lake, G. Klimeck, R. C. Bowen, and D. Jovanovic, "Single and Multiband Modeling of Quantum Electron Transport Through Layered Semiconductor Devices," J.Appl.Phys., vol. 81, no. 12, pp. 7845-7869, 1997.

[34] M. Sabathil, S. Hackenbuchner, J. A. Majewski, G. Zandler, and P. Vogl, "Towards Fully Quantum Mechanical 3D Device Simulations," J.Computational Electronics, vol. 1, pp. 81-85, 2002.

[35] D. Mamaluy, M. Sabathil, and P. Vogl, "Efficient Method for the Calculation of Ballistic Quantum Transport," J.Appl.Phys., vol. 93, no. 8 , pp. 4628-4633, 2003.

[36] J. Guo and M. Lundstrom, "Role of Phonon Scattering in Carbon Nanotube Field-Effect Transistors," Appl.Phys.Lett., vol. 86, pp. 193 103-1193 103-2, 2005

[37] A. Javey, J. Guo, D. Farmer, Q. Wang, E. Yenilmez, R. Gordon, M. Lundstrom, and H. Dai, "Self-Aligned Ballistic Molecular Transistors and Electrically Parallel Nanotube Arrays," Nano Letters, vol. 4, no. 7, pp. 1319-1322, 2004

[38] X. Shao and Z. Yu, "Nanoscale FinFET Simulations: A Quasi-3D Quantum Mechanical Model Using NEGF," Solid-State Electron., vol. 49, pp. $1435-1445,2005$

[39] C. Jungemann, N. Subba, J.-S. Goo, C. Riccobene, Q. Xiang, and B. Meinerzhagen, "Investigation of Strained Si/SiGe Devices by MC Simulation," Solid-State Electron., vol. 48, no. 8, pp. 1417-1422, 2004.

[40] M. Fischetti, "Theory of Electron Transport in Small Semiconductor Devices Using the Pauli Master Equation,” J.Appl.Phys., vol. 83, no. 1, pp. 270-291, 1998.

[41] P. Palestri, D. Esseni, S. Eminente, C. Fiegna, E. Sangiorgi, and L. Selmi, "Understanding Quasi-Ballistic Transport in Nano-MOSFETs: Part I - Scattering in the Channel, and in the Drain," IEEE Trans.Electron Devices, vol. 52, no. 12, pp. 2727-2735, 2005.

[42] M. Gilbert, R. Akis, and D. Ferry, "Phonon-Assisted Ballistic to Diffusive Crossover in Silicon Nanowire Transistors," J.Appl.Phys., vol. 98, no. 9, pp. 094303-1-8, 2005.

[43] A. Svizhenko and M. P. Anantram, "Role of Scattering in Nanotransistors," IEEE Trans.Electron Devices, vol. 50, pp. 1459-1466, 2003

[44] M. Pourfath, H. Kosina, B. Cheong, W. Park, and S. Selberherr, "Improving DC and AC Characteristics of Ohmic Contact Carbon Nanotube Fiel Effect Transistors," in Proc. European Solid-State Device Research Conf., 2005, pp. 541-544.

[45] H. Kosina, M. Nedjalkov, and S. Selberherr, "A Monte Carlo Method Seamlessly Linking Quantum and Classical Transport Calculations," J. Computational Electronics, vol. 2, no. 2-4, pp. 147-151, 2002.

[46] A. Wettstein, A. Schenk, and W. Fichtner, "Quantum Device-Simulation with the Density-Gradient Model on Unstructured Grids," IEEE Trans.Electron Devices, vol. 48, no. 2, pp. 279-284, 2001.

[47] T. Hoehr, A. Schenk, A. Wettstein, and W. Fichtner, "On DensityGradient Modeling of Tunneling Through Insulators," in Proc. Intl. Conf. on Simulation of Semiconductor Processes and Devices, 2002, pp. 275278.

[48] L. Shifren, C. Ringhofer, and D. Ferry, "A Wigner Function-Based Quantum Ensemble Monte Carlo Study of a Resonant Tunneling Diode," IEEE Trans.Electron Devices, vol. 50, no. 3, pp. 769-773, 2003.

[49] V. Sverdlov, A. Gehring, H. Kosina, and S. Selberherr, "Quantum Transport in Ultra-Scaled Double-Gate MOSFETs: A Wigner FunctionBased Monte Carlo Approach," Solid-State Electron., vol. 49, no. 9, pp. 1510-1515, 2005. 